\title{
Global Approximations to Solutions of Initial Value Problems*
}

\author{
By Luis Kramarz
}

\begin{abstract}
A wide class of implicit one-step methods for the construction of global approximations to solutions of initial value problems is studied. Approximations more general than piecewise polynomials can be constructed to exploit certain characteristics of the differential equation. Error bounds are given for the general class of methods but emphasis is placed on methods based on Hermite interpolation, for which higher rates of convergence are obtained for special choices of interpolation points. Computational examples are presented.
\end{abstract}

1. Introduction. In recent years there has been an interest in the construction of piecewise polynomial approximations to the solutions of ordinary differential equations, with the benefit of derivative approximations. We present here a wide class of implicit one-step methods for the construction of more general global approximations to the solution of a single initial value problem of order $s$. The methods are based on families of linear operators, not necessarily projections, which satisfy certain conditions; they include as special cases the collocation schemes of Hulme [10], [11], Russell and Shampine [19], de Boor and Swartz [7], and several of the more general projection methods of Wittenbrink [28], as applied to initial value problems. Several schemes such as the Newton-Cotes block methods of Watts and Shampine [26], some of whose global aspects were investigated by Williams and de Hoog [27], are equivalent to collocation; we point out here that so are the methods of Callender [5] and of Micula [17]. Other methods also included are the natural spline block methods of Andria, Byrne and Hill [2].

Our main theory on existence, convergence and error bounds for the approximate solutions is based mostly on the collectively compact operator theory of Anselone and Moore [3]. We present several families of operators which can be used to produce specific methods, and emphasize the methods based on Hermite interpolation, for which we obtain higher rates of convergence along the lines of de Boor and Swartz [7]. Numerical examples are given.

Many of the main results we obtained will carry over to systems of initial value problems and boundary value problems since they can be set up as integral equations having essentially the same properties as the integral equations considered here.

Received December 6, 1976.

AMS (MOS) subject classifications (1970). Primary 65L05.

Key words and phrases. Initial value problems, ordinary differential equations, one-step implicit methods, global approximations, collocation, projection methods, piecewise polynomials, spline.

* This research was supported in part by the U. S. Army Research Office under Grant DA-AG29-76-G-0261.

Copyright $\odot$ 1978, American Mathematical Society 
2. The Problem and Its Approximation. Consider the initial value problem

$$
\begin{gathered}
y^{(s)}(x)=f\left(x, y(x), \ldots, y^{(m)}(x)\right), \quad 0 \leqslant x \leqslant b, m \leqslant s-1, \\
y^{(i)}(0)=g_{i}, \quad 0 \leqslant i \leqslant s-1,
\end{gathered}
$$

where $f$ is a real-valued function continuous in $D=[0, b] \times R^{m+1}$, with $R=(-\infty$, $\infty)$. We will assume that $(2.1),(2.2)$ has a unique solution $y \in C^{s}[0, b]$, which is the case if, for example, $f$ satisfies a uniform Lipschitz condition in $D$.

Let $a_{k}, 0 \leqslant k \leqslant m$, be arbitrary constants and define the operator $L: C^{s}[0, b]$ $\rightarrow C[0, b]$ by

$$
(L u)(x)=u^{(s)}(x)-\sum_{k=0}^{m} a_{k} u^{(k)}(x)
$$

Equation (2.1) is equivalent to $(L y)(x)=w(x) H(x ; y)$, where $w$ and $H$ are any two functions satisfying

$$
w(x) H(x ; y)=f(x ; y)-\sum_{k=0}^{m} a_{k} y^{(k)}(x),
$$

and we use the notation $g(x ; y)=g\left(x, y(x), \ldots, y^{(m)}(x)\right)$. Let $G(x, t)=v(x-t)$, $0 \leqslant t \leqslant x \leqslant b$, where $v$ is the solution of $L v=0, v^{(i)}(0)=0,0 \leqslant i \leqslant s-2, v^{(s-1)}(0)$ $=1$. Then the solution $y$ of $(2.1),(2.2)$ satisfies

$$
y(x)=F(x)+\int_{0}^{x} G(x, t) w(t) H(t ; y) d t, \quad 0 \leqslant x \leqslant b,
$$

where $F(x)=\Sigma_{k=1}^{s} \alpha_{k} \varphi_{k}(x)$ is the solution of $L u=0$ subject to (2.2). $G$ and the $\varphi_{k}$ can be easily constructed since $L$ has constant coefficients.

Equation (2.3) is set in the Banach space $C^{s-1}[0, b]$, where $C^{r}[0, b]$ denotes the space of all $r$-times continuously differentiable functions in $[0, b]$, with norm

$$
\|g\|_{r}=\sup _{0<x<b} \sum_{i=0}^{r}\left|g^{(i)}(x)\right| .
$$

The approximate methods will consist of replacing (2.3) by a perturbed equation, also set in $C^{s-1}[0, b]$, which in turn is equivalent to an algebraic system of equations. Let $\left\{\Delta_{n}\right\}$ be a sequence of partitions of $[0, b]$

$$
\Delta_{n}: 0=x_{n, 0}<x_{n, 1}<\cdots<x_{n, n}=b .
$$

For convenience, we will write $x_{n, i}$ as $x_{i}$.

Let $\left|\Delta_{n}\right| \equiv \max \Delta x_{j} \rightarrow 0$ as $n \rightarrow \infty$, where $\Delta x_{j}=x_{j}-x_{j-1}$. We say that $\left\{\Delta_{n}\right\}$ is quasi-uniform if $\max \left|\Delta_{n}\right| / \Delta x_{j} \leqslant A$ for some constant $A$.

Define $C_{\Delta_{n}}^{r}[0, b]$ as the set of all real-valued functions $g$ on $[0, b]$ such that $g \in C^{r}\left(x_{j-1}, x_{j}\right), 1 \leqslant j \leqslant n$, and such that $g^{(i)}\left(x_{j-1}^{+}\right)$and $g^{(i)}\left(x_{j}^{-}\right)$exist for $0 \leqslant i \leqslant r$, $1 \leqslant j \leqslant n$. Also define for $g \in C_{\Delta_{n}}^{r}[0, b]$ 


$$
\|g\|_{r, n}=\max _{1<j<n} \sup _{x_{j-1}<x<x_{j}} \sum_{i=0}^{r}\left|g^{(i)}(x)\right| .
$$

The approximate solution $y_{n}$ to the solution $y$ of (2.3) is defined by

$$
y_{n}(x)=F_{n}(x)+\int_{0}^{x} G(x, t) w(t) P_{n}\left(H\left(\cdot ; y_{n}\right)\right)(t) d t, \quad 0 \leqslant x \leqslant b,
$$

where $F_{n}(x)=\Sigma_{k=1}^{s} \alpha_{k}^{n} \varphi_{k}(x)$, and $\alpha_{k}^{n}$ is an approximation to $\alpha_{k}$. The operators $\left\{P_{n}\right\}$ are a family of linear operators satisfying

(2.6a) $P_{n}: C^{q}[0, b] \rightarrow C_{\Delta_{n}}^{0}[0, b]$ for some nonnegative integer $q$ independent of $n$;

(2.6b) $\left\|P_{n} g\right\|_{0, n} \leqslant C\|g\|_{q}$ for all $g \in C^{q}[0, b]$, where $C$ is independent of $n$;

$$
\left\|P_{n} g-g\right\|_{0, n} \rightarrow 0 \text { as } n \rightarrow \infty \text { for each } g \in C^{q}[0, b] \text {. }
$$

Even though the analysis will be carried out for Eqs. (2.3) and (2.5), in practice the solution $y_{n}$ of $(2.5)$ can be found in a step-by-step process since for $j=1,2$, ..., $n$,

$$
y_{n}(x)=F_{n, j}(x)+\int_{x_{j-1}}^{x} G(x, t) w(t) P_{n}\left(H\left(\because ; y_{n}\right)\right)(t) d t, \quad x_{j-1} \leqslant x \leqslant x_{j},
$$

where $F_{n, j}(x)=\Sigma_{k=1}^{s} \alpha_{k, j}^{n} \varphi_{k}(x)$ is the solution of $L u=0, u^{(i)}\left(x_{j-1}\right)=y_{n}^{(i)}\left(x_{j-1}\right)$, $0 \leqslant i \leqslant s-1$.

In operator notation we write (2.3) and (2.5) as

$$
\begin{gathered}
(I-K) y=F \\
\left(I-K_{n}\right) y_{n}=F_{n},
\end{gathered}
$$

where

$$
\begin{aligned}
(K u)(x) & =\int_{0}^{x} G(x, t) w(t)(T u)(t) d t, \\
\left(K_{n} u\right)(x) & =\int_{0}^{x} G(x, t) w(t)\left(P_{n} T u\right)(t) d t
\end{aligned}
$$

with $(T u)(x)=H(x ; u)$.

We will assume that $w \in C[0, b]$ and that $H \in C^{2}(N)$, where

$$
\begin{aligned}
N=\left\{\left(x, z_{0}, z_{1}, \ldots, z_{m}\right): 0 \leqslant x \leqslant b,\left|z_{k}-y^{(k)}(x)\right|\right. & \leqslant \delta, \\
0 & \leqslant k \leqslant m, \delta>0\}
\end{aligned}
$$

is a neighborhood of the exact solution $y$.

3. Preliminaries. In order to use a variation of a Kantorovich theorem which appears in [3], we will need the following results.

LEMMA 3.1. Let $K$ be the operator of (2.10) and suppose $w \in C[0, b]$ and $H \in C^{2}(N)$. Then $K: C^{s-1}[0, b] \rightarrow C^{s-1}[0, b]$ has first and second Fréchet derivatives at $y$ given by 


$$
\begin{gathered}
\left(K^{\prime}(y) u\right)(x)=\int_{0}^{x} G(x, t) w(t)\left(T^{\prime}(y) u\right)(t) d t \\
\left(K^{\prime \prime}(y) u v\right)(x)=\int_{0}^{x} G(x, t) w(t)\left(T^{\prime \prime}(y) u v\right)(t) d t,
\end{gathered}
$$

where

$$
\begin{aligned}
\left(T^{\prime}(y) u\right)(t) & =\sum_{i=0}^{m} \frac{\partial H(t ; y)}{\partial z_{i}} u^{(i)}(t) \quad \text { and } \\
\left(T^{\prime \prime}(y) u v\right)(t) & =\sum_{i=0}^{m} \sum_{k=0}^{m} \frac{\partial^{2} H(t ; y)}{\partial z_{k} \partial z_{i}} v^{(k)}(t) u^{(i)}(t)
\end{aligned}
$$

Proof. The expressions for $T^{\prime}(y)$ and $T^{\prime \prime}(y)$ are well known for $T: C^{s-1}[0, b]$ $\rightarrow C[0, b]$. The expressions for $K^{\prime}(y)$ and $K^{\prime \prime}(y)$ follow from their definition.

Lemma 3.2. Let $K_{n}$ be defined by (2.11), where $w \in C[0, b], P_{n}$ satisfies (2.6a)-(2.6c), and $H \in C^{q+2}(N)$. Then $K_{n}: C^{s-1}[0, b] \rightarrow C^{s-1}[0, b]$ has first and second Fréchet derivatives at y given by

$$
\begin{aligned}
& \left(K_{n}^{\prime}(y) u\right)(x)=\int_{0}^{x} G(x, t) w(t)\left(P_{n} T^{\prime}(y) u\right)(t) d t, \\
& \left(K_{n}^{\prime \prime}(y) u v\right)(x)=\int_{0}^{x} G(x, t) w(t)\left(P_{n} T^{\prime \prime}(y) u v\right)(t) d t,
\end{aligned}
$$

with $T^{\prime}(y), T^{\prime \prime}(y)$ as in Lemma 3.1.

Proof. One can show [15] that $T^{\prime}(y)$ and $T^{\prime \prime}(y)$ are unchanged when $T$ is considered as an operator from $C^{s-1}[0, b]$ into $C^{q}[0, b]$. The results follow from $(2.6 \mathrm{~b})$ and the definition of the derivatives.

The following lemmas are required to show that $\left\|\left(K^{\prime}(y)-K_{n}^{\prime}(y)\right) K_{n}^{\prime}(y)\right\|_{s-1} \rightarrow$ 0 as $n \stackrel{\prime}{\rightarrow} \infty$, which is a basic requirement in the next section. To simplify the notation, we let $c_{k}(x)=\partial H(x ; y) / \partial z_{k}, 0 \leqslant k \leqslant m$, and we will use $C$ as a generic constant throughout. The next result is found in [8, pp. 344-345].

Lemma 3.3. A subset $A$ of $C^{s-1}[0, b]$ has compact closure if and only if $A$ is bounded and for every $\epsilon>0$ there exists $\delta>0$ such that for all $u, v \in[0, b]$ with $|u-v|<\delta$ and all $g \in A$, it is true that $\left|g^{(s-1)}(u)-g^{(s-1)}(v)\right|<\epsilon$.

LEMмA 3.4. If $w, c_{k} \in C[0, b]$ then the operator $K^{\prime}(y)$ of $(3.1)$ is a compact linear operator from $C^{s-1}[0, b]$ into $C^{s-1}[0, b]$.

Proof. Let $B=\left\{z \in C^{s-1}[0, b]:\|z\|_{s-1} \leqslant 1\right\}$. If $g \in K^{\prime}(y) B$ then for some $z \in B$,

$$
\|g\|_{s-1}=\left\|K^{\prime}(y) z\right\|_{s-1} \leqslant \sup _{0<x<b} \sum_{i=0}^{s-1} \sum_{k=0}^{m} \int_{0}^{b}\left|\frac{\partial^{i} G(x, t)}{\partial x^{i}} w(t) c_{k}(t)\right| d t,
$$

so $K^{\prime}(y) B$ is bounded. Now let $\epsilon>0, u, v \in[0, b]$. Then with an application of the Mean Value Theorem, there is some $\xi$ between $u$ and $v$ such that 


$$
\begin{aligned}
& \left|g^{(s-1)}(u)-g^{(s-1)}(v)\right|=\left|\left(K^{\prime}(y) z\right)^{(s-1)}(u)-\left(K^{\prime}(y) z\right)^{(s-1)}(v)\right| \\
& \quad=\left|\int_{0}^{u} \frac{\partial^{s} G(\xi, t)}{\partial x^{s}}(u-v) w(t)\left(T^{\prime}(y) z\right)(t) d t-\int_{u}^{v} \frac{\partial^{s-1} G(v, t)}{\partial x^{s-1}} w(t)\left(T^{\prime}(y) z\right)(t) d t\right| \\
& \quad \leqslant C|u-v|
\end{aligned}
$$

where $C$ is independent of $u, v$ and $g$. The hypotheses of Lemma 3.3 are immediate; and therefore, $K^{\prime}(y)$ is compact.

LEMMA 3.5. Let $\left\{P_{n}\right\}$ be a sequence of linear operators satisfying (2.6a)-(2.6c), and let $w \in C[0, b], H \in C^{q+1}(N)$, with $0 \leqslant q \leqslant s-m-1$. Then the sequence of operators $\left\{K_{n}^{\prime}(y)\right\}$ given in (3.3) satisfies

(i) $K_{n}^{\prime}(y): C^{s-1}[0, b] \rightarrow C^{s-1}[0, b]$;

(ii) $\left\|K_{n}^{\prime}(y) g-K^{\prime}(y) g\right\|_{s-1} \rightarrow 0$ as $n \rightarrow \infty$ for any $g \in C^{s-1}[0, b]$;

(iii) $\left\{K_{n}^{\prime}(y)\right\}$ is collectively compact, i.e., $B^{*}=\bigcup_{n=1}^{\infty} K_{n}^{\prime}(y) B$, where $B$ is the unit ball in $C^{s^{-1}}[0, b]$, has compact closure.

Proof. Property (i) is clear. Let $g \in B^{*}$. Then $g=K_{n}^{\prime}(y) z$ for some $n$ and some $z \in B$. Hence

$$
\begin{aligned}
\|g\|_{s-1} & =\left\|K_{n}^{\prime}(y) z\right\|_{s-1}=\sup _{0<x<b} \sum_{i=0}^{s-1}\left|\int_{0}^{x} \frac{\partial^{i} G(x, t)}{\partial x^{i}} w(t)\left(P_{n} T^{\prime}(y) z\right)(t) d t\right| \\
& \leqslant\left\|P_{n} T^{\prime}(y) z\right\|_{0, n} \sup _{0<x<b} \sum_{i=0}^{s-1} \int_{0}^{x}\left|\frac{\partial^{i} G(x, t)}{\partial x^{i}} w(t)\right| d t \leqslant C\left\|T^{\prime}(y) z\right\|_{q} \leqslant C,
\end{aligned}
$$

where $C$ is independent of $n$ and $g$. The last two inequalities follow from (2.6b), $0 \leqslant$ $q \leqslant s-m-1$ and $\|z\|_{s-1} \leqslant 1$. Now let $\epsilon>0, u, v \in[0, b]$. Then, much as in the proof of Lemma 3.4,

$$
\left|g^{(s-1)}(u)-g^{(s-1)}(v)\right| \leqslant C|u-v|\left\|P_{n} T^{\prime}(y) z\right\|_{0, n} \leqslant C|u-v|\left\|T^{\prime}(y) z\right\|_{q} \leqslant C|u-v|,
$$

where $C$ is independent of $u, v$ and $g$. By Lemma 3.3, $B^{*}$ has compact closure. To complete the proof, let $h \in C^{s-1}[0, b]$. Then

$$
\left\|K_{n}^{\prime}(y) h-K^{\prime}(y) h\right\|_{s-1} \leqslant C\left\|P_{n} T^{\prime}(y) h-T^{\prime}(y) h\right\|_{0, n},
$$

where $C$ is independent of $n$. Since $T^{\prime}(y) h \in C^{q}[0, b]$, (ii) follows from (2.6c).

The next theorem is a collection of several results of Anselone [3, Theorem 1.6, Corollary 1.9], on which we will base the main results of this section.

THEOREM 3.1. Let $X$ be a Banach space and $K: X \rightarrow X$ be a compact linear operator such that $(I-K)^{-1}$ exists. Let $\left\{K_{n}\right\}$ be a sequence of linear operators satisfying

(i) $K_{n}: X \rightarrow X$;

(ii) $\left\|K_{n} g-K g\right\| \rightarrow 0$ as $n \rightarrow \infty$ for each $g \in X$;

(iii) $\left\{K_{n}\right\}$ is collectively compact.

Then

1) $\left\|\left(K_{n}-K\right) K_{n}\right\| \rightarrow 0$ and $\left\|\left(K_{n}-K\right) K\right\| \rightarrow 0$ as $n \rightarrow \infty$; 
2) there exists $N>0$ such that for all $n \geqslant N$, the operators $\left(I-K_{n}\right)^{-1}$ exist and are uniformly bounded.

Lemma 3.6. Assume all the hypotheses of Lemma 3.4. Then $\left(I-K^{\prime}(y)\right)^{-1}$ exists and is a bounded operator from $C^{s-1}[0 ; b]$ onto $C^{s-1}[0, b]$.

Proof. Since $K^{\prime}(y)$ is compact, by the Fredholm alternative $\left(I-K^{\prime}(y)\right)^{-1}$ exists if and only if $\left(I-K^{\prime}(y)\right) C^{s-1}[0, b]=C^{s-1}[0, b]$, in which case $\left(I-K^{\prime}(y)\right)^{-1}$ is bounded. Let $v \in C^{s-1}[0, b]$. We will show $\left(I-K^{\prime}(y)\right) u=v$ has a solution. Let $z$ be the solution of the initial value problem $L z=w T^{\prime}(y) z+w T^{\prime}(y) v, z^{(i)}(0)=0,0 \leqslant$ $i \leqslant s-1$. Then $z$ satisfies $z=K^{\prime}(y) z+K^{\prime}(y) v$ and $u=z+v$ satisfies $\left(I-K^{\prime}(y)\right) u$ $=v$.

4. Existence and Convergence of the Approximate Solutions.

TheOREM 4.1 [3, Theorem 6.5]. Let $S$ be an operator on a Banach space $X$, $z \in X$ and $R x \equiv(I-S) x-z$, where $I$ is the identity operator. Let $M$ and $(I-M)^{-1}$ be bounded linear operators on $X$, and $x_{0} \in X$. Suppose $S^{\prime}\left(x_{0}\right)$ is compact and

$$
\begin{array}{cc}
\left\|(I-M)^{-1}\right\| \leqslant \beta, \quad\left\|R x_{0}\right\| \leqslant d, & \left\|\left(M-S^{\prime}\left(x_{0}\right)\right) R x_{0}\right\| \leqslant d_{0}, \\
\left\|\left(M-S^{\prime}\left(x_{0}\right)\right) S^{\prime}\left(x_{0}\right)\right\| \leqslant d_{1}<\frac{1}{\beta}, & \left\|S^{\prime}(u)-S^{\prime}(v)\right\| \leqslant \gamma\|u-v\| \\
\text { on } B\left(x_{0}, r\right) \equiv\left\{u:\left\|u-x_{0}\right\| \leqslant r\right\}, & \text { on } B\left(x_{0}, r\right), \\
\left.\left\|\left(M-S^{\prime}\left(x_{0}\right)\right)\left(S^{\prime}(u)-S^{\prime}(v)\right)\right\| \leqslant d_{2}\|u-v\| \quad d_{0}\right) & r_{0}=\frac{\beta\left(d+d_{0}\right) w^{*}(h)}{1-\beta d_{1}} \leqslant r,
\end{array}
$$

where $w^{*}(h) \equiv\left(1-(1-2 h)^{1 / 2}\right) / h$ for $0<h \leqslant 1 / 2$, and $w^{*}(0)=1$.

Then there exists a unique $x^{*} \in B\left(x_{0}, r_{0}\right)$ such that $R x^{*}=0$. The Newton iterates $x_{i}$ are defined in $B\left(x_{0}, r_{0}\right)$ and converge to $x^{*}$.

The hext theorem is the most important of this section and is a consequence of Theorem 4.1.

THEOREM 4.2. Consider Eqs. (2.8) and (2.9) and let $\left\{P_{n}\right\}$ satisfy (2.6a)-(2.6c). Also let $w \in C[0, b]$ and $H \in C^{q+2}(N)$, where $q$ is an integer such that $0 \leqslant q \leqslant s$ - $m$ - 1. Suppose (2.1), (2.2) has a unique solution y. Finally, let

$$
\max _{1<k<s}\left\{\left|\alpha_{k}^{n}-\alpha_{k}\right|\right\} \rightarrow 0 \text { as } n \rightarrow \infty
$$

Then there is some $N>0$ such that for all $n \geqslant N$ there exists $r_{n}$ such that Eq. (2.9) has a unique solution $y_{n} \in B\left(y, r_{n}\right) \equiv\left\{z \in C^{s-1}[0, b]:\|z-y\|_{s-1} \leqslant r_{n}\right\}$. The Newton iterates $y_{n, i}$ are defined in $B\left(y, r_{n}\right)$ for $n \geqslant N$ and converge to $y_{n}$. In addition there is a constant $C$ independent of $n$ such that

$$
\begin{aligned}
\left\|y-y_{n}\right\|_{s-1} & \leqslant C\left\|F-F_{n}+K y-K_{n} y\right\|_{s-1} \\
& \leqslant C\left[\left\|\sum_{k=1}^{s}\left(\alpha_{k}^{n}-\alpha_{k}\right) \varphi_{k}\right\|_{s-1}+\left\|P_{n} H(\cdot ; y)-H(\cdot ; y)\right\|_{0, n}\right] .
\end{aligned}
$$


Proof. By Lemma $3.6\left(I-K^{\prime}(y)\right)^{-1}$ exists and is a bounded linear operator on $C^{s-1}[0, b]$. Let $\left\|\left(I-K^{\prime}(y)\right)^{-1}\right\| \leqslant \beta$. Also, by Lemmas 3.4 and $3.5, K^{\prime}(y)$ and $\left\{K_{n}^{\prime}(y)\right\}$ satisfy the hypothesis of Theorem 3.1 , hence $\left\|\left(K^{\prime}(y)-K_{n}^{\prime}(y)\right) K_{n}^{\prime}(y)\right\|_{s-1} \rightarrow$ 0 as $n \rightarrow \infty$. Let $R_{n} u \equiv\left(I-K_{n}\right) u-F_{n}$. Then there is a constant $C$ independent of $n$ such that

$$
\begin{aligned}
\left\|R_{n} y\right\|_{s-1} & =\left\|F-F_{n}+K y-K_{n} y\right\|_{s-1} \\
& \leqslant C\left[\left\|\sum_{k=1}^{s}\left(\alpha_{k}^{n}-\alpha_{k}\right) \varphi_{k}\right\|_{s-1}+\left\|P_{n} H(\cdot ; y)-H(\cdot ; y)\right\|_{0, n}\right],
\end{aligned}
$$

and $\left\|\left(K^{\prime}(y)-K_{n}^{\prime}(y)\right) R_{n} y\right\|_{s-1} \leqslant C\left\|R_{n} y\right\|_{s-1}$. The last inequality is a consequence of the pointwise convergence of $K_{n}^{\prime}(y)$ to $K^{\prime}(y)$ and the Banach-Steinhaus theorem. For $u, v \in B(y, \delta)$ let $w_{t}=v+t(u-v) ; \delta$ is as in (2.12). Then by the Mean Value

Theorem we have

$$
\left\|K_{n}^{\prime}(u)-K_{n}^{\prime}(v)\right\|_{s-1} \leqslant\|u-v\|_{s-1} \sup _{0<t<1}\left\|K_{n}^{\prime \prime}\left(w_{t}\right)\right\|_{s-1} .
$$

But if $\left\|u_{1}\right\|_{s-1} \leqslant 1,\left\|v_{1}\right\|_{s-1} \leqslant 1$, then $\left\|K_{n}^{\prime \prime}\left(w_{t}\right) u_{1} v_{1}\right\|_{s-1} \leqslant C\left\|P_{n} T^{\prime \prime}\left(w_{t}\right) u_{1} v_{1}\right\|_{0, n} \leqslant$ $C\left\|T^{\prime \prime}\left(w_{t}\right) u_{1} v_{1}\right\|_{q} \leqslant C$, where $C$ is independent of $t, u_{1}, v_{1}$, and $n$, since $w_{t} \in B(y, \delta)$ and the partial derivatives of $H$ are uniformly bounded in the region $N$. Thus, $\left\|K_{n}^{\prime}(u)-K_{n}^{\prime}(v)\right\|_{s-1} \leqslant C_{1}\|u-v\|_{s-1}$ for all $u, v \in B(y, \delta)$, with $C_{1}$ independent of $n$. We also have that $\left\|\left(K^{\prime}(y)-K_{n}^{\prime}(y)\right)\left(K_{n}^{\prime}(u)-K_{n}^{\prime}(v)\right)\right\|_{s-1} \leqslant C_{2}\|u-v\|_{s-1}$ for all $u, v \in$ $B(y, \delta)$, with $C_{2}$ independent of $n$. Now choose $N$ large enough that for $n \geqslant N$

$$
\begin{aligned}
& d_{n} \equiv\left\|\left(K^{\prime}(y)-K_{n}^{\prime}(y)\right) K_{n}^{\prime}(y)\right\|_{s-1} \leqslant \frac{1}{2 \beta}, \\
& h_{n} \equiv \frac{\beta^{2}\left[\left\|\left(K^{\prime}(y)-K_{n}^{\prime}(y)\right) R_{n} y\right\|_{s-1}+\left\|R_{n} y\right\|_{s-1}\right]\left(C_{1}+C_{2}\right)}{\left(1-\beta d_{n}\right)^{2}} \leqslant \frac{1}{2},
\end{aligned}
$$

and

$$
r_{n} \equiv \frac{\beta\left[\left\|\left(K^{\prime}(y)-K_{n}^{\prime}(y)\right) R_{n} y\right\|_{s-1}+\left\|R_{n} y\right\|_{s-1}\right] w^{*}\left(h_{n}\right)}{\left(1-\beta d_{n}\right)} \leqslant \delta .
$$

By Theorem 4.1 we conclude that for $n \geqslant N$, the equation $\left(I-K_{n}\right) y_{n}-F_{n}=0$ has a unique solution $y_{n} \in B\left(y, r_{n}\right)$ and the Newton iterates converge to $y_{n}$. The error bounds are derived from $\left\|y-y_{n}\right\|_{s-1} \leqslant r_{n} \leqslant C\left\|R_{n} y\right\|_{s-1}$, where $C$ is independent of $n$.

Corollary 4.1. Assume all the hypotheses of Theorem 4.2 and let $\alpha_{k}^{n}$ in (2.5) be chosen so that $F_{n}$ is the solution of $L u=0, u^{(i)}(0)=g_{i}^{n}, 0 \leqslant i \leqslant s-1$, where

$$
\epsilon_{n}=\max _{0<i<s-1}\left\{\left|y^{(i)}(0)-g_{i}^{n}\right|\right\} \rightarrow 0 \quad \text { as } n \rightarrow \infty
$$

Then there is a constant $C$ independent of $n$ such that

$$
\left\|y-y_{n}\right\|_{s-1} \leqslant C\left(\epsilon_{n}+\left\|P_{n} H(\cdot ; y)-H(\cdot ; y)\right\|_{0, n}\right) .
$$


In particular, if $w \equiv 1$, then

$$
\left\|y-y_{n}\right\|_{s-1} \leqslant C\left(\epsilon_{n}+\left\|P_{n} L y-L y\right\|_{0, n}\right) .
$$

Proof. By construction, $F-F_{n}$ is the solution of $L u=0, u^{(i)}(0)=y^{(i)}(0)-$ $g_{i}^{n}, 0 \leqslant i \leqslant s-1$. Hence $\max _{k}\left\{\left|\alpha_{k}-\alpha_{k}^{n}\right|\right\} \leqslant C \epsilon_{n}$, where $C$ is independent of $n$. The results now follow from the theorem and since $L y=H(\cdot ; y)$ if $w \equiv 1$.

COROLlary 4.2. Assume all the hypotheses of Corollary 4.1. Then $\left\|y_{n}-y\right\|_{s-1} \rightarrow 0$ as $n \rightarrow \infty$.

Proof. The result is obvious from Corollary 4.1 and (2.6c).

If Eq. (2.1) is linear, we can simplify the theory and weaken some of the hypotheses leading to Corollary $4.1[15]$.

5. Construction of Sequences of Operators $P_{n}$. Before we can discuss the transformation of Eq. (2.7) into an equivalent system of algebraic equations, we will introduce families of operators $P_{n}$ which will give rise to specific methods. It is possible to construct operators $P_{n}$ satisfying (2.6a)-(2.6c) starting with an operator $Q$ defined on $C^{q}[0,1]$. Define the norm $\|\cdot\|$ by

$$
\|G\|=\sup _{0<t<1}|G(t)|
$$

and let $\omega(G, h)=\sup \{|G(u)-G(v)|:|u-v| \leqslant h\}$ be the modulus of continuity of a function $G$ defined on $[0,1]$.

Lemma 5.1. Suppose $Q$ is an operator defined on $C^{q}[0,1]$ satisfying

(i) $(Q G)(t)=\Sigma_{i=0}^{s_{1}} \lambda_{i}(G) p_{i}(t), 0 \leqslant t \leqslant 1, s_{1}$ a positive integer, where $\left\{p_{i}\right\}_{0}^{s_{1}}$ is a basis for a subspace $X_{1}$ of $C[0,1]$, and $\left\{\lambda_{i}\right\}_{0}^{s_{1}}$ is a set of real bounded linear functionals defined on $C^{q}[0,1]$, independent over $X_{1}$;

(ii) there are constants $C$, $\alpha$ such that for all $G \in C^{q}[0,1]$,

$$
\|Q G-G\| \leqslant C \omega\left(G^{(q)}, \alpha\right) .
$$

If $g \in C^{q}[0, b]$, let

$$
\left(P_{n} g\right)(x)=\left(Q G_{j}\right)\left(\frac{x-x_{j-1}}{\Delta x_{j}}\right), \quad x_{j-1}<x<x_{j}, 1 \leqslant j \leqslant n,
$$

with $G_{j}(t)=g\left(x_{j-1}+t \Delta x_{j}\right)$, and let $\left(P_{n} g\right)\left(x_{j}\right), 0 \leqslant j \leqslant n$, be the average of the leftand right-hand limits. Then $\left\{P_{n}\right\}$ satisfies (2.6a)-(2.6c).

Proof. The conclusion follows in a straightforward manner from the definition of $\boldsymbol{P}_{\boldsymbol{n}}$. For example,

$$
\begin{aligned}
\sup _{x_{j-1}<x<x_{j}}\left|\left(P_{n} g-g\right)(x)\right| & =\sup _{0<t<1}\left|\left(Q G_{j}-G_{j}\right)(t)\right| \leqslant C \omega\left(G_{j}^{(q)}, \alpha\right) \\
& \leqslant C\left(\Delta x_{j}\right)^{q} \omega\left(g^{(q)}, \alpha \Delta x_{j}\right) \leqslant C \omega\left(g^{(q)}, \alpha\left|\Delta_{n}\right|\right),
\end{aligned}
$$

where $C$ is independent of $n$. Hence (2.6c) is a result of the continuity of $g^{(q)}$ and since $\left|\Delta_{n}\right| \longrightarrow 0$ as $n \rightarrow \infty$. 
Example 5.1. Hermite Interpolation. Consider a partition $D_{0}$ of $[0,1]$ given by

$$
D_{0}: 0 \leqslant \gamma_{1}<\gamma_{2}<\cdots<\gamma_{p} \leqslant 1, \quad p \geqslant 2 .
$$

Let $r_{i}$ be a nonnegative integer, $1 \leqslant i \leqslant p$, and let $q=\max r_{i}$. For $G \in C^{q}[0,1]$ define $Q_{1} G$ to be the polynomial of degree $\leqslant \tilde{p}-1$ such that

$$
\left(Q_{1} G\right)^{(i)}\left(\gamma_{k}\right)=G^{(i)}\left(\gamma_{k}\right), \quad 1 \leqslant k \leqslant p, 0 \leqslant i \leqslant r_{k},
$$

where $\tilde{p}=p+\Sigma_{i=1}^{p} r_{i}$. We can write

$$
\left(Q_{1} G\right)(t)=\sum_{k=1}^{p} \sum_{i=0}^{r_{k}} G^{(i)}\left(\gamma_{k}\right) l_{k, i}(t), \quad 0 \leqslant t \leqslant 1,
$$

where $l_{k, i}$ is a polynomial of degree $\leqslant \tilde{p}-1$ and $l_{k, i}^{(v)}\left(\gamma_{u}\right)=\delta_{i v} \delta_{k u}, 1 \leqslant u \leqslant p, 0 \leqslant$ $v \leqslant r_{u}$. Here $\delta_{i v}$ is the Kronecker delta. An explicit expression for $l_{k, i}$ is found in [22]. If we let

$$
\gamma_{i j}=x_{j-1}+\gamma_{i} \Delta x_{j}, \quad 1 \leqslant i \leqslant p, 1 \leqslant j \leqslant n,
$$

then

$$
\left(P_{n} g\right)(x)=\sum_{k=1}^{p} \sum_{i=0}^{r_{k}}\left(\Delta x_{j}\right)^{i} g^{(i)}\left(\gamma_{k j}\right) l_{k, i}\left(\frac{x-x_{j-1}}{\Delta x_{j}}\right), \quad x_{j-1}<x<x_{j}
$$

We have been unable to find in the literature error bounds of the form (ii) of Lemma 5.1 for general Hermite interpolation. Most bounds given assume that $G \in$ $C^{\tilde{p}}[0,1]$; Birkhoff, Schultz and Varga [4] give error bounds for the case $p=2$, but they require $G^{(q)}$ to be absolutely continuous. We will derive our error bounds based on the following results of Jackson [14, pp. 15-17].

THEOREM 5.1. If $G \in C[0,1]$, then for each $N=1,2,3, \ldots$, there exists $a$ polynomial $p_{N}$ of degree $\leqslant N$ such that $\left\|G-p_{N}\right\| \leqslant C \omega(G, 1 / N)$, where $C$ is independent of $N$ and $G$. If $G \in C^{v}[0,1]$ for some $v \geqslant 1$, then for each $N>v-1$ there exists a polynomial $p_{N}$ of degree $\leqslant N$ such that $\left\|G-p_{N}\right\| \leqslant C\left\|G^{(v)}\right\| / N^{v}$, where $C$ is independent of $N$ and $G$.

LEMmA 5.2. If $G \in C^{q}[0,1]$, then

$$
\left\|\left(Q_{1} G-G\right)^{(i)}\right\| \leqslant C \omega\left(G^{(q)}, 1 /(\tilde{p}-q-1)\right), \quad 0 \leqslant i \leqslant q,
$$

where $C$ is independent of $G$. If $G \in C^{(q+u)}[0,1]$ for some $u$ such that $1 \leqslant u \leqslant \tilde{p}$ $-q-1$, then

$$
\left\|\left(Q_{1} G-G\right)^{(i)}\right\| \leqslant C\left\|G^{(q+u)}\right\|, \quad 0 \leqslant i \leqslant q
$$

where $C$ is independent of $G$.

Proof. Suppose $q \geqslant 1$ and that $r_{k}=q$. Define an operator $Q_{0}$ on $C[0,1]$ as follows: if $z \in C[0,1]$, let $\left(Q_{0} z\right)(t)=\left(Q_{1} S(z ; t)\right)^{(q)}(t)$, where

$$
S(z ; t)=\int_{\gamma_{k}}^{t} \frac{(t-u)^{q-1}}{(q-1) !} z(u) d u, \quad 0 \leqslant t \leqslant 1 .
$$


One verifies from this definition that for any $G \in C^{q}[0,1]$,

$$
Q_{0}\left(G^{(q)}\right)=\left(Q_{1} G\right)^{(q)}
$$

and also that $Q_{0} v=v$ for any polynomial $v$ of degree $\leqslant \tilde{p}-q-1$. Hence, $\left(Q_{1} G-G\right)^{(q)}(t)=v(t)-G^{(q)}(t)-\left(Q_{1} S\left(v-G^{(q)} ; t\right)\right)^{(q)}$. However, there is a constant $C$ independent of $G$ and $v$ such that $\left\|\left(Q_{1} S\left(v-G^{(q)} ; \cdot\right)\right)^{(q)}\right\| \leqslant C\left\|v-G^{(q)}\right\|$. Therefore, $\left\|\left(Q_{1} G-G\right)^{(q)}\right\| \leqslant C\left\|v-G^{(q)}\right\|$ for any polynomial $v$ of degree $\leqslant \tilde{p}-q-$ 1. This inequality and Theorem 5.1 imply the conclusion of the lemma for $i=q$. For $0 \leqslant i \leqslant q-1$, the conclusions follow from

$$
\left(Q_{1} G-G\right)^{(q-i-1)}(t)=\int_{\gamma_{k}}^{t}\left(Q_{1} G-G\right)^{(q-i)}(u) d u .
$$

Finally, if $q=0$, we have $\left\|Q_{1} G-G\right\|=\left\|v-G+Q_{1}(v-G)\right\| \leqslant C\|v-G\|$ for any polynomial of degree $\leqslant \tilde{p}-1$, where $C$ is independent of $G$ and $v$. The rest of the proof is again an application of Theorem 5.1.

It will be useful to have error bounds as in Lemma 5.2 but for derivatives higher than $q$.

Lemma 5.3. If $G \in C^{\tilde{p}}[0,1]$, then

$$
\left\|\left(Q_{1} G-G\right)^{(i)}\right\| \leqslant C\left\|G^{(\tilde{p})}\right\|, \quad 0 \leqslant i \leqslant \tilde{p}-1,
$$

where $C$ is independent of $G$.

Proof. By Rolle's theorem, $\left(Q_{1} G\right)^{(i)}$ is a Hermite interpolant of degree $\tilde{p}-i-$ 1 of $G^{(i)}$ in $[0,1], 0 \leqslant i \leqslant \tilde{p}-1$. Since $G^{(i)} \in C^{\tilde{p}-i}[0,1]$, then by the standard error bound for Hermite interpolation [12, p. 256] we have the result of the lemma.

Lemмa 5.4. Let $\left\{P_{n}\right\}$ be the sequence of operators obtained from $Q_{1}$. Then there is a constant $C$ independent of $n$ such that

(i) for all $g \in C^{q}[0, b]$,

$$
\sup _{x_{j-1}<x<x_{j}}\left|\left(P_{n} g-g\right)(x)\right| \leqslant C\left(\Delta x_{j}\right)^{q} \omega\left(g^{(q)}, \Delta x_{j} /(\tilde{p}-q-1)\right) ;
$$

(ii) for all $g \in C^{q+u}[0, b]$, where $1 \leqslant u \leqslant \tilde{p}-q-1$,

$$
\sup _{x_{j-1}<x<x_{j}}\left|\left(P_{n} g-g\right)^{(i)}(x)\right| \leqslant C\left(\Delta x_{j}\right)^{q+u-i} \sup _{x_{j-1}<x<x_{j}}\left|g^{(q+u)}(x)\right|, \quad 0 \leqslant i \leqslant q
$$

(iii) for all $g \in C^{\tilde{p}}[0, b]$,

$$
\sup _{x_{j-1}<x<x_{j}}\left|\left(P_{n} g-g\right)^{(i)}(x)\right| \leqslant C\left(\Delta x_{j}\right)^{\tilde{p}-i} \sup _{x_{j-1}<x<x_{j}}\left|g^{(\tilde{p})}(x)\right|, \quad 0 \leqslant i \leqslant \tilde{p}-1 .
$$

Proof. The proof follows from definition (5.1) and Lemmas 5.2 and 5.3. The above error bounds will be combined with Corollary 4.1 to obtain bounds for $y-y_{n}$.

Example 5.2. Natural Spline Interpolation. Let $\left\{\boldsymbol{\gamma}_{i}\right\}_{0}^{p}$ be a uniform partition of $[0,1]$, where $\gamma_{i}=i / p, 0 \leqslant i \leqslant p, p \geqslant 1$. If $l$ is an integer such that $1 \leqslant l \leqslant p+1$, let $S_{2 l-1}$ be the set of natural splines of degree $2 l-1$ having the knots $\gamma_{i}$, i.e., $v \in$ $S_{2 l-1}$ if $v \in C^{2 l-2}[0,1]$, if $v$ is a polynomial of degree $\leqslant 2 l-1$ in each subinterval 
$\left(\gamma_{i}, \gamma_{i+1}\right)$, and for $l>1$, if $v^{(k)}\left(\gamma_{i}\right)=0, l \leqslant k \leqslant 2 l-2, i=0, p$. Define $Q_{2}$ : $C[0,1] \rightarrow S_{2 l-1}$ by

$$
\left(Q_{2} G\right)(t)=\sum_{i=0}^{p} G\left(\gamma_{i}\right) T_{i}(t), \quad 0 \leqslant t \leqslant 1,
$$

where $T_{i} \in S_{2 l-1}$ and $T_{i}\left(\gamma_{j}\right)=\delta_{i j}$. As described in [20], $Q_{2} G$ is well defined; it is also a $g$-spline type II interpolate of $G$ studied in [21], but the error bounds given there are not in the form we require.

Lemma 5.5. Suppose $1<l \leqslant p+1$. Then if $G \in C[0,1]$,

$$
\left\|Q_{2} G-G\right\| \leqslant C \omega(G, 1 /(l-1))
$$

where $C$ is independent of $G$. If also $G \in C^{u}[0,1]$ for some $u$ such that $1 \leqslant u \leqslant l$ -1 , then $\left\|Q_{2} G-G\right\| \leqslant C\left\|G^{(u)}\right\|$, where $C$ is independent of $G$. Moreover, if $G \in$ $C^{l}[0,1]$, then

$$
\left\|\left(Q_{2} G-G\right)^{(i)}\right\| \leqslant\left\|G^{(l)}\right\|, \quad 0 \leqslant i \leqslant l-1 .
$$

Proof. It is clear that $Q_{2} v=v$ for every polynomial $v$ of degree $\leqslant l-1$. Hence, $\left\|Q_{2} G-G\right\|=\left\|Q_{2}(G-v)-(G-v)\right\| \leqslant C\|G-v\|$, for some $C$ independent of $G$. The first two inequalities of the lemma follow from Theorem 5.1. We obtain the final inequality adapting some ideas of [1]. Let $x \in[0,1]$; then in $l-1$ consecutive subintervals containing $x$ there is some $\xi_{x}^{i}$ such that $\left(Q_{2} G-G\right)^{(i)}\left(\xi_{x}^{i}\right)=0,0 \leqslant i \leqslant l-1$, by Rolle's theorem. Hence,

$$
\left|\left(Q_{2} G-G\right)^{(l-1)}(x)\right|=\left|\int_{\xi_{x}^{l-1}}^{x}\left(Q_{2} G-G\right)^{(l)}(t) d t\right| \leqslant\left[\int_{0}^{1}\left[\left(Q_{2} G-G\right)^{(l)}(t)\right]^{2} d t\right]^{1 / 2}
$$

and in general,

$$
\left\|\left(Q_{2} G-G\right)^{(i)}\right\| \leqslant\left[\int_{0}^{1}\left[\left(Q_{2} G-G\right)^{(l)}(t)\right]^{2} d t\right]^{1 / 2}, \quad 0 \leqslant i \leqslant l-1 .
$$

But by the first integral relation for $g$-splines (see for example [21, Theorem 16]),

$$
\left\|\left(Q_{2} G-G\right)^{(i)}\right\| \leqslant\left[\int_{0}^{1}\left[G^{(l)}(t)\right]^{2} d t\right]^{1 / 2} \leqslant\left\|G^{(l)}\right\|, \quad 0 \leqslant i \leqslant l-1 .
$$

It is straightforward to construct error bounds such as those of Lemma 5.4 for the operators $P_{n}$ derived from $Q_{2}$.

Example 5.3. Local Spline Approximating Operators. As an example where the operator $Q$ is not necessarily a projection, we will use a subclass of the explicit spline operators of Lyche and Schumaker [16, Example 3.4]. Let $D_{3}: 0=\gamma_{0}<\gamma_{1}$ $<\cdots<\gamma_{p}=1$ be a partition of $[0,1]$, and $k$ be an integer, $k \geqslant 1$. Let $S_{k}$ be the set of smooth polynomial splines of degree $k-1$ with simple knots at $\gamma_{i}$. Extend $D_{3}$ to a nondecreasing sequence $\left\{\gamma_{i}\right\}_{1-k}^{p+k-1}$, with $\gamma_{i}<\gamma_{i+k}$. Fix an integer $l, 1 \leqslant l$ $\leqslant k$, and for each $i=1-k, \ldots, p-1$, let $\left\{t_{i u}\right\}_{u=1}^{l}$ be distinct numbers in $[0,1]$ 
$\cap\left[\gamma_{i}, \gamma_{i+k}\right]$. The operator $Q_{3}: C[0,1] \rightarrow S_{k}$ is defined by

$$
\left(Q_{3} G\right)(t)=\sum_{i=1-k}^{p-1} \sum_{u=1}^{l} a_{i u} G\left[t_{i 1}, \ldots, t_{i u}\right] N_{i, k}(t), \quad 0 \leqslant t \leqslant 1
$$

where $G\left[a_{0}, a_{1}, \ldots, a_{r}\right]$ denotes the $r$ th order divided difference of $G$. Here $N_{i, k}(x)$ $=\left(\gamma_{i+k}-\gamma_{i}\right) G_{k}(\because x)\left[\gamma_{i}, \ldots, \gamma_{i+k}\right], 1-k \leqslant i \leqslant p-1$, with $G_{k}(t ; x)=(t-x)_{+}^{k-1}$, $a_{i 1}=1$ and

$a_{i u}=\sum_{v=0}^{u-1}\left((-1)^{v} \operatorname{sym}_{v}\left(t_{i 1}, \ldots, t_{i u-1}\right) \operatorname{sym}_{u-1-v}\left(\gamma_{i+1}, \ldots, \gamma_{i+k-1}\right) /\left(\begin{array}{c}k-1 \\ u-1-v\end{array}\right)\right)$,

$$
2 \leqslant u \leqslant l
$$

where $\operatorname{sym}_{i}\left(x_{1}, \ldots, x_{r}\right)$ is defined implicitly by

$$
\prod_{i=1}^{r}\left(x+x_{i}\right)=\sum_{v=1}^{r+1} \operatorname{sym}_{r-v+1}\left(x_{1}, \ldots, x_{r}\right) x^{v-1} .
$$

The error bounds that we need are readily obtained from [16, Theorem 5.3]

Lemma 5.6. Suppose $1 \leqslant l \leqslant k$. If $G \in C[0,1]$, then there are constants $C, \alpha$ independent of $G$ such that $\left\|Q_{3} G-G\right\| \leqslant C \omega(G, \alpha)$. If $G \in C^{u}[0,1]$ for some $u$ such that $1 \leqslant u \leqslant l$, then $\left\|\left(Q_{3} G-G\right)^{(i)}\right\| \leqslant C\left\|G^{(u)}\right\|, 0 \leqslant i \leqslant k-1$.

Bounds such as those of Lemma 5.4 can be easily obtained for the operators $\boldsymbol{P}_{n}$ derived from $Q_{3}$.

Example 5.4. Moments. Let $Q_{4}$ be an operator defined on $C[0,1]$ given by $\left(Q_{4} G\right)(t)=\Sigma_{i=0}^{s_{1}} \lambda_{i}(G) p_{i}(t), 0 \leqslant t \leqslant 1$, where

$$
\lambda_{i}(G)=\int_{0}^{1} t^{i} G(t) d t, \quad 0 \leqslant i \leqslant s_{1},
$$

and $p_{i}$ is a polynomial of degree $\leqslant s_{1}$ with $\lambda_{i}\left(p_{k}\right)=\delta_{i k}$. Since $Q_{4} v=v$ for all polynomials $v$ of degree $\leqslant s_{1}$, we can use Theorem 5.1 as before to obtain results such as those of Lemmas 5.2, 5.3 and 5.4.

6. Numerical Solution of the Approximate Problem. The Newton iterates for the solution of Eq. (2.9) are of the form

$$
y_{n, r+1}(x)=F_{n}(x)+\int_{0}^{x} G(x, t) w(t) P_{n}\left(H_{n, r}\left(\cdot ; y_{n, r+1}\right)\right)(t) d t, \quad 0 \leqslant x \leqslant b,
$$

where $H_{n, r}\left(x ; y_{n, r+1}\right)=\left(T y_{n, r}-T^{\prime}\left(y_{n, r}\right)\left(y_{n, r}-y_{n, r+1}\right)\right)(x), r=0,1,2, \ldots$. For each fixed $r$, the solution of (6.1) can be found step-by-step, solving at each step

$$
y_{n, r+1}(x)=F_{n, j}^{r}(x)+\int_{x_{j-1}}^{x} G(x, t) w(t) P_{n}\left(H_{n, r}\left(\cdot ; y_{n, r+1}\right)\right)(t) d t
$$

$$
x_{j-1} \leqslant x \leqslant x_{j}
$$

where $F_{n, j}^{r}$ is the solution of $L u=0, u^{(i)}\left(x_{j-1}\right)=y_{n, r+1}^{(i)}\left(x_{j-1}\right), 0 \leqslant i \leqslant s-1$.

In practice, however, we iterate with Eq. (6.2) until we obtain $y_{n}$ to a desired 
accuracy in each subinterval before proceeding to the next. An extrapolation of $y_{n}$ to the next subinterval can be taken as initial estimate $y_{n, 0}$.

If $P_{n}$ is defined by (5.1), then Eq. (6.2) implies that $y_{n, r+1}$ has the form

$$
y_{n, r+1}(x)=F_{n, j}^{r}(x)+\sum_{i=0}^{s_{1}} b_{i j}^{r} d_{i j}(x), \quad x_{j-1} \leqslant x \leqslant x_{j}
$$

where

$$
d_{i j}(x)=\int_{x_{j-1}}^{x} G(x, t) w(t) p_{i}\left(\frac{t-x_{j-1}}{\Delta x_{j}}\right) d t, \quad 0 \leqslant i \leqslant s_{1}, 1 \leqslant j \leqslant n .
$$

Notice that $F_{n, j}^{r}$ is known explicitly in $\left[x_{j-1}, x_{j}\right]$ and that $d_{i j}(x)$ can also be found explicitly if $w \equiv 1$ and $p_{i}$ is a piecewise polynomial, which is usually the case. Operating with $L$ on both sides of (6.2) and (6.3), subtracting the resulting equations and assuming that $w$ has at most a countable number of zeros in $[0, b]$ we find that the $b_{i j}^{r}$ satisfy the linear system

$$
b_{i j}^{r}=\lambda_{i}\left(H_{n, r}\left(x_{j-1}+(\cdot) \Delta x_{j} ; y_{n, r+1}\right)\right), \quad 0 \leqslant i \leqslant s_{1} .
$$

Conversely, each solution of (6.5) determines a solution of (6.2) through (6.3). One can write $(6.5)$ in the form

$$
b_{i j}^{r}-\sum_{u=0}^{s_{1}} b_{u j}^{r} z_{i u}=w_{i}, \quad 0 \leqslant i \leqslant s_{1}
$$

where $z_{i u}=O\left(\left|\Delta_{n}\right|^{s-m}\right)$, since $\partial^{k} G(x, t) / \partial x^{k}$ has a zero of multiplicity $s-1-k$ at $x=t$; hence (6.6) has a unique solution for $n$ sufficiently large which could be obtained by iteration.

If the functionals $\lambda_{i}$ are derivative evaluations at points in $[0,1]$ (up to the $s-$ $m-1$ th derivative), there is an alternate approach to the solution of (6.3). From (6.3) and (6.5) we have

$$
y_{n, r+1}(x)=F_{n, j}^{r}(x)+\sum_{i=0}^{s_{1}} \lambda_{i}\left(H_{n, r}\left(x_{j-1}+(\cdot) \Delta x_{j} ; y_{n, r+1}\right)\right) d_{i j}(x),
$$

$$
0 \leqslant i \leqslant s_{1}
$$

If the value $y_{n, r+1}^{(v)}\left(\gamma_{k j}\right)$ appears on the right-hand side of (6.7), one differentiates both sides of (6.7) $v$ times and evaluates at $\gamma_{k j}$, obtaining a system of linear equations for these values; the size of this system is no bigger than that of (6.5) if $\boldsymbol{P}_{n}$ is as in Example 5.1 and $m=0$.

Without referring to Newton's method we see from (2.7) that $y_{n}$ is of the form

$$
y_{n}(x)=F_{n, j}(x)+\sum_{i=0}^{s_{1}} b_{i j} d_{i j}(x), \quad x_{j-1} \leqslant x \leqslant x_{j} .
$$


Then an argument like that leading to (6.5) gives the nonlinear system

$$
b_{i j}=\lambda_{i}\left(H\left(x_{j-1}+(\cdot) \Delta x_{j} ; y_{n}\right)\right), \quad 0 \leqslant i \leqslant s_{1} .
$$

If $w \equiv 1$ and $P_{n}$ is obtained from a projection $Q$, then (2.7) and (5.1) imply $\left(L y_{n}\right)\left(x_{j-1}+t \Delta x_{j}\right)=Q\left(H\left(x_{j-1}+(\cdot) \Delta x_{j} ; y_{n}\right)\right)(t), 0<t<1$. Operating with $Q$ on both sides of this equation (interpreting the left-hand side as defined in all of $[0,1]$ by its limiting values) we obtain after some simplification

$$
\lambda_{i}\left(y_{n}^{(s)}\left(x_{j-1}+(\cdot) \Delta x_{j}\right)\right)=\lambda_{i}\left(f\left(x_{j-1}+(\cdot) \Delta x_{j} ; y_{n}\right)\right), \quad 0 \leqslant i \leqslant s_{1} .
$$

Equation (6.9) refers directly to the original differential equation. If $\boldsymbol{P}_{\boldsymbol{n}}$ is as in Example 5.1, then (6.9) becomes

$$
y_{n}^{(s+v)}\left(\gamma_{k j}\right)=\left(f\left(\because y_{n}\right)\right)^{(v)}\left(\gamma_{k j}\right), \quad 1 \leqslant k \leqslant p, 0 \leqslant v \leqslant r_{k}, 1 \leqslant j \leqslant n
$$

If in addition each $a_{k}=0$, then $y_{n} \in C^{s-1}[0, b]$ is a polynomial of degree $\leqslant \tilde{p}+s$ -1 in $\left[x_{j-1}, x_{j}\right]$ whose coefficients can be found from $(6.10)$ and the continuity requirements.

7. An Application of the Theory. The next theorem illustrates how one can combine Corollary 4.1 and bounds such as those of Lemma 5.4 to obtain useful results about specific methods.

THEOREM 7.1. Consider the initial value problem (2.1), (2.2) and suppose it has a solution $y$. Let $q$ be an integer satisfying $0 \leqslant q \leqslant s-m-1$, and $\left\{\Delta_{n}\right\}$ a sequence of partitions of $[0, b]$ given by (2.4) with $\left|\Delta_{n}\right| \rightarrow 0$. Also, let $\left\{P_{n}\right\}$ be the sequence of linear operators of Example 5.1. Suppose $w \in C[0, b]$ and $H \in C^{q+2}(N)$, with $N$ as in (2.12). In addition, let $\epsilon_{n} \leqslant C\left|\Delta_{n}\right|^{m_{1}}$, where $\epsilon_{n}$ is defined in (4.1) and $C$ and $m_{1}$ are independent of $n$, and let $\alpha_{k}^{n}$ in (2.5) be chosen as in Corollary 4.1. Then there is some $N>0$ such that for all $n \geqslant N$ there exists $r_{n} \leqslant \delta$ such that Eq. (2.9) has a unique solution $y_{n} \in B\left(y, r_{n}\right)=\left\{z \in C^{s-1}[0, b]:\|z-y\|_{s-1} \leqslant r_{n}\right\}$; the Newton iterates $y_{n, i}$ are defined in $B\left(y, r_{n}\right)$ for $n \geqslant N$ and converge to $y_{n}$; there is a constant $C$ independent of $n$ such that for $n \geqslant N$,

$$
\sup _{0<x<b}\left|\left(y-y_{n}\right)^{(i)}(x)\right| \leqslant C\left|\Delta_{n}\right|^{\min \left(m_{1}, q+2\right)}, \quad 0 \leqslant i \leqslant s-1 .
$$

If in addition $H(\cdot ; y) \in C^{q+u}[0, b]$ for some $u$ satisfying $3 \leqslant u \leqslant \tilde{p}-q$, then

$$
\sup _{0<x<b}\left|\left(y-y_{n}\right)^{(i)}(x)\right| \leqslant C\left|\Delta_{n}\right|^{\min \left(m_{1}, q+u\right)}, \quad 0 \leqslant i \leqslant s-1 .
$$

In particular, if $u=\tilde{p}-q, w \in C^{\tilde{p}-1}[0,1]$ and $\left\{\Delta_{n}\right\}$ is quasi-uniform, then

$$
\max _{1<j<n} \sup _{x_{j-1}<x<x_{j}}\left|\left(y-y_{n}\right)^{(s+i)}(x)\right| \leqslant C\left|\Delta_{n}\right|^{\min \left(m_{1}, \tilde{p}\right)-i}, \quad 0 \leqslant i \leqslant \tilde{p}-1 .
$$

Proof. All results except (7.3) are a consequence of Corollary 4.1 and Lemma 5.4. To obtain (7.3), apply $L$ to both sides of (2.3) and (2.5), subtract the resulting equations and differentiate $i$ times. Write the equation as 


$$
\left(y-y_{n}\right)^{(s+i)}(x)=\left[w\left(H(\cdot ; y)-P_{n} H(\cdot ; y)\right)\right]^{(i)}(x)+\left[w P_{n}\left(H(\cdot ; y)-H\left(\cdot ; y_{n}\right)\right)\right]^{(i)}(x)
$$

$$
+\sum_{k=0}^{m} a_{k}\left(y-y_{n}\right)^{(k+i)}(x), \quad x_{j-1}<x<x_{j}, 0 \leqslant i \leqslant \tilde{p}-1
$$

We have that

$$
\sup _{x_{j-1}<x<x_{j}}\left|\left(H\left(\cdot ; y_{n}\right)-H(\cdot ; y)\right)^{(u)}(x)\right|=O\left(\left|\Delta_{n}\right|^{\min \left(\tilde{p}, m_{1}\right)}\right), \quad 0 \leqslant u \leqslant q .
$$

To see this, define $R_{1}(g)(x)=g\left(x ; y_{n}\right)-g(x ; y)$ for any function $g \in C^{q+1}(N)$. By the Mean Value Theorem and (7.2) it follows that for $i=0$,

$$
\sup _{x_{j-1}<x<x_{j}}\left|\left(R_{1}(g)\right)^{(i)}(x)\right|=O\left(\left|\Delta_{n}\right|^{\min \left(\tilde{p}, m_{1}\right)}\right)
$$

Now

$\left(R_{1}(g)\right)^{\prime}(x)=R_{1}\left(\frac{\partial g}{\partial x}\right)(x)+\sum_{i=0}^{m} R\left(\frac{\partial g}{\partial z_{i}}\right)(x) y^{(i+1)}(x)+\sum_{i=0}^{m} \frac{\partial g}{\partial z_{i}}\left(x ; y_{n}\right)\left(y_{n}-y\right)^{(i+1)}(x)$

Hence again by the Mean Value Theorem, (7.2) and by (7.6) with $i=0$, we obtain (7.6) for $i=1$. In general, differentiating $R_{1}(g)$ up to $q$ times and using a uniform bound on all the partial derivatives up to order $q+1$ of $g$ in $N$ we obtain (7.6) for $0 \leqslant i \leqslant q$, since $m+q \leqslant s-1$. Hence (7.5) is satisfied.

By Lemma 5.4 the first term in the right-hand side of $(7.4)$ is $O\left(\mid \Delta_{n} \tilde{p}^{\tilde{p}-i}\right)$. By (7.5) and (5.4), the second term is $O\left(\left|\Delta_{n}\right|^{\min \left(\tilde{p}, m_{1}\right)-i}\right)$. Combining these results with (7.2), we obtain (7.3).

If Eq. (2.1) is linear, a theorem such as Theorem 7.1 can be obtained [15] if $H(x ; y) \in C^{q}[0, b]$ only. If $f(x ; y)=r(x)+\sum_{k=0}^{m} b_{k}(x) y^{(k)}(x)$, then we write instead of (2.3),

$$
\begin{aligned}
y(x)= & \sum_{k=1}^{s} \alpha_{k} \varphi_{k}(x)+\int_{0}^{x} G(x, t) r(t) d t \\
& +\int_{0}^{x} G(x, t) w_{1}(x) H_{1}(t ; y) d t, \quad 0 \leqslant x \leqslant b .
\end{aligned}
$$

where $H_{1}(x ; y)=\Sigma_{k=0}^{m} c_{k}(x) y^{(k)}(x)$ and $w_{1}(x) c_{k}(x)=b_{k}(x)-a_{k}, 0 \leqslant k \leqslant m$. The approximate equation is now

$$
\begin{aligned}
y_{n}(x)= & \sum_{k=1}^{s} \alpha_{k}^{n} \varphi_{k}(x)+\int_{0}^{x} G(x, t)\left(P_{n} r\right)(t) d t \\
& +\int_{0}^{x} G(x, t) w_{1}(t) P_{n}\left(H_{1}\left(\cdot ; y_{n}\right)\right)(t) d t, \quad 0 \leqslant x \leqslant b .
\end{aligned}
$$


If $n$ is sufficiently large, we find that (7.8) has a unique solution; and that if $w \in$ $C[0, b]$ and $r, c_{k} \in C^{q}[0, b]$, then

$$
\sup _{0<x<b}\left|\left(y-y_{n}\right)^{(i)}(x)\right|
$$

$$
\begin{array}{r}
\leqslant C\left(\epsilon_{n}+\left|\Delta_{n}\right|^{q}\left(\omega\left(r^{(q)}, \Delta_{n} /(\tilde{p}-q-1)\right)+\omega\left(H_{1}^{(q)}, \Delta_{n} /(\tilde{p}-q-1)\right)\right)\right) \\
0 \leqslant i \leqslant s-1 .
\end{array}
$$

If in addition $r, H_{1}(\because y) \in C^{q+u}[0, b]$ for some $u$ such that $1 \leqslant u \leqslant \tilde{p}-q$, then (7.2) is satisfied, and so is (7.3) if $u=\tilde{p}-q, w_{1} \in C^{\tilde{p}-1}[0, b]$ and $\left\{\Delta_{n}\right\}$ is quasiuniform.

8. Higher Rates. When the operators of Example 5.1 are used as in the preceding section, it is possible to improve the rates of convergence for the lower derivatives, especially at the partition points, if we can choose the points $\gamma_{k} \in[0,1]$ so that

$$
\int_{0}^{1} \prod_{k=1}^{p}\left(t-\gamma_{k}\right)^{1+r_{k}} v(t) d t=0
$$

for every polynomial of degree $\leqslant r$, where $r$ is some integer satisfying $r \leqslant \tilde{p}-1$. To obtain the higher rates we rely on a basic idea of de Boor and Swartz [7], modified to account for $w$ and the fact that $y_{n}$ is not necessarily a piecewise polynomial.

LemmA 8.1. Assume all the hypotheses of Theorem 7.1, but let $g_{i}^{n}=y^{(i)}(0)$, $0 \leqslant i \leqslant s-1$. In addition, suppose $H \in C^{m_{2}}(N)$ and $w \in C^{m_{3}}[0, b]$, where $m_{2}=$ $\max \{\tilde{p}, \tilde{p}+r+1+m-s\}, m_{3}=\max \{0, \tilde{p}+r+1+m-s\}$ and $r$ is some nonnegative integer. Then for all $n$ sufficiently large, the equation $\left(I-K_{n}^{\prime}(y)\right) R_{n} y=F_{n}+$ $K_{n} y-K_{n}^{\prime}(y) y$ has a unique solution $R_{n} y$, where $K_{n}, F_{n}$ are as in the proof of Theorem 7.1. Also,

$$
\sup _{x_{j-1}<x<x_{j}}\left|\left(y-R_{n} y\right)^{(i)}(x)\right| \leqslant C\left(\frac{\left|\Delta_{n}\right|}{\Delta x_{j}}\right)^{\tilde{p}}, \quad 0 \leqslant i \leqslant \tilde{p}+r+1+m,
$$

where $C$ is independent of $n$ and

$$
\left(y_{n}-y\right)^{(i)}(x)=\left(R_{n} y-y\right)^{(i)}(x)+O\left(\left|\Delta_{n}\right|^{2} \tilde{p}\right), \quad 0 \leqslant x \leqslant b, 0 \leqslant i \leqslant s-1
$$

Proof. By Lemmas 3.4, 3.5, 3.6 and Theorem 3.1, $\left(I-K_{n}^{\prime}(y)\right)^{-1}$ exists and is uniformly bounded for $n$ sufficiently large. By Theorem 7.1, Eq. (2.9) has a unique solution $y_{n} \in B\left(y, r_{n}\right)$ for $n$ sufficiently large. We can write $y_{n}=F_{n}+K_{n} y_{n}=F_{n}$ $+K_{n} y+K_{n}^{\prime}(y)\left(y_{n}-y\right)+E\left(y_{n}\right)$, where by a form of the second Mean Value Theorem there is a constant $C$ independent of $n$ such that

$$
\left\|E\left(y_{n}\right)\right\|_{s-1} \leqslant C\left\|y_{n}-y\right\|_{s-1}^{2} .
$$

$$
\text { Hence, } y_{n}=\left(I-K_{n}^{\prime}(y)\right)^{-1}\left(F_{n}+K_{n} y-K_{n}^{\prime}(y) y+E\left(y_{n}\right)\right) \text { and so }
$$

$$
y_{n}-y=R_{n} y-y+\left(I-K_{n}^{\prime}(y)\right)^{-1} E\left(y_{n}\right) \text {. }
$$


By (7.2) and (8.4), Eq. (8.5) implies

$$
\left\|\left(y_{n}-y\right)-\left(R_{n} y-y\right)\right\|_{s-1} \leqslant C\left\|y-y_{n}\right\|_{s-1}^{2} \leqslant C\left|\Delta_{n}\right|^{2 \tilde{p}} .
$$

Rewriting (8.5) and taking the $i$ th derivative, we have by (2.6b) and (8.6) that

$$
\begin{aligned}
\left(y_{n}-y\right)^{(i)}(x)-\left(R_{n} y-y\right)^{(i)}(x) & \leqslant C\left\|T^{\prime}(y)\left(\left(y_{n}-y\right)-\left(R_{n} y-y\right)\right)\right\|_{q}+\left\|E\left(y_{n}\right)\right\|_{s-1} \\
& \leqslant C\left|\Delta_{n}\right|^{2 \tilde{p}}, \quad 0 \leqslant x \leqslant b, 0 \leqslant i \leqslant s-1 .
\end{aligned}
$$

Therefore, (8.3) is satisfied. Now by (7.2) and (8.3),

$$
\sup _{x_{j-1}<x<x_{j}}\left|\left(R_{n} y-y\right)^{(i)}(x)\right|=O\left(\mid \Delta_{n} \tilde{p}\right), \quad 0 \leqslant i \leqslant s-1 .
$$

If $\tilde{p}+r+1+m-s<0$, then (8.7) implies (8.2). So suppose $\tilde{p}+r+1+m-s$ $\geqslant 0$. $R_{n} y$ satisfies

$$
L\left(R_{n} y\right)(x)=w(x) P_{n}\left[T y-T^{\prime}(y)\left(y-R_{n} y\right)\right](x), \quad x_{j-1}<x<x_{j} .
$$

Subtracting $L y=w T y$ from (8.8), we obtain

$$
\begin{aligned}
\left(y-R_{n} y\right)^{(s+i)}(x)=[ & \left.w\left(T y-P_{n} T y\right)\right]^{(i)}(x)+\left[w P_{n}\left(T^{\prime}(y)\left(y-R_{n} y\right)\right)\right]^{(i)}(x) \\
+ & +\sum_{k=0}^{m} a_{k}\left(y-R_{n} y\right)^{(k+i)}(x) \\
& x_{j-1}<x<x_{j}, 0 \leqslant i \leqslant \tilde{p}+r+1+m-s .
\end{aligned}
$$

If $0 \leqslant i \leqslant \tilde{p}-1$, then by Lemma 5.4 and (8.7), the first term in the right-hand side of (8.9) is $O\left(\mid \Delta_{n} \tilde{p}^{\tilde{p}-i}\right)$, and the second term is $O\left(\left|\Delta_{n}\right|^{\tilde{p}} /\left(\Delta x_{j}\right)^{i}\right)$. Therefore,

$$
\begin{aligned}
\sup _{x_{j-1}<x<x_{j}}\left|\left(R_{n} y-y\right)^{(s+i)}(x)\right| & =O\left(\left|\Delta_{n}\right| \tilde{p} /\left(\Delta x_{j}\right)^{i}\right) \\
& =O\left(\mid \Delta_{n} \tilde{p} /\left(\Delta x_{j}\right)^{\tilde{p}-1}\right), \quad 0 \leqslant i \leqslant \tilde{p} .
\end{aligned}
$$

If $\tilde{p} \leqslant i \leqslant \tilde{p}+r+1+m-s$, then by (8.7), $(8.10)$ and since $\left(P_{n} g\right)^{(i)} \equiv 0$ for any $g \in C^{q}[0, b]$, Eq. (8.9) implies

$$
\begin{aligned}
\sup _{x_{j-1}<x<x_{j}}\left|\left(y-R_{n} y\right)^{(s+i)}(x)\right|=O\left(\left|\Delta_{n}\right| \tilde{p} /\left(\Delta x_{j}\right)^{\tilde{p}}\right), & \\
& \tilde{p} \leqslant i \leqslant \tilde{p}+r+1+m-s .
\end{aligned}
$$

The result (8.2) is a consequence of (8.7), (8.10) and (8.11).

The next theorem, with $a_{k}=0$ and $w \equiv 1$, contains the results, specialized to (2.1), (2.2), of [7, Theorem 4.1], and also those of [28, Theorem 5] whenever $0 \leqslant$ $q \leqslant s-m-1$. 
THEOREM 8.1. Assume all the hypotheses of Theorem 7.1 and suppose the $\gamma_{k}$ satisfy Eq. (8.1) for some $r \leqslant \tilde{p}-1$. Suppose in addition that $g_{i}^{n}=y^{(i)}(0), 0 \leqslant i \leqslant$ $s-1$, and that $H \in C^{m} 4(N), w \in C^{m}[0, b]$, where $m_{4}=\tilde{p}+r+2$ and $m_{5}=$ $\max \{\tilde{p}+r+1+m-s, r+1\}$. Then the approximations $y_{n}$ of Theorem 7.1 also satisfy for $n \geqslant N$,

$$
\max _{0<j<n}\left|\left(y-y_{n}\right)^{(i)}\left(x_{j}\right)\right| \leqslant C\left|\Delta_{n}\right|^{\tilde{p}+r+1}, \quad 0 \leqslant i \leqslant s-1,
$$

and

$$
\max _{0<x<b}\left|\left(y-y_{n}\right)^{(i)}(x)\right| \leqslant C\left|\Delta_{n}\right|^{\tilde{p}+\min (r+1, s-i)}, \quad 0 \leqslant i \leqslant s-1,
$$

where $C$ is independent of $n$.

Proof. Let $N$ in Theorem 7.1 be sufficiently large so that the conclusions of Lemma 8.1 are valid for $n \geqslant N$. Also let $E(x, t)=u_{t}(x), 0 \leqslant t \leqslant x$, where $u_{t}(x)$ is the solution of $\left(L-w T^{\prime}(y)\right) v=0, v^{(i)}(t)=0,0 \leqslant i \leqslant s-2, v^{(s-1)}(t)=1$. Then since $\left(y-R_{n} y\right)^{(i)}(0)=0,0 \leqslant i \leqslant s-1$, we have

$$
\begin{aligned}
\left(y-R_{n} y\right)(x) & =\int_{0}^{x} E(x, t)\left(L-w T^{\prime}(y)\right)\left(y-R_{n} y\right)(t) d t \\
& =\int_{0}^{x} E(x, t) w(t) v_{n}(t) d t, \quad 0 \leqslant x \leqslant b,
\end{aligned}
$$

with

$$
\begin{aligned}
v_{n}(x) & =\frac{1}{w(x)}\left(L-w T^{\prime}(y)\right)\left(y-R_{n} y\right)(x) \\
& =\left[T y-P_{n} T y-T^{\prime}(y)\left(y-R_{n} y\right)+P_{n}\left(T^{\prime}(y)\left(y-R_{n} y\right)\right)\right](x),
\end{aligned}
$$

$x_{j-1}<x<x_{j}$. The last equality follows from (8.8) and $L y=w T y$. Hence if $E_{i}(x, t)$ $=\partial^{i} E(x, t) / \partial x^{i}$

(8.13) $\left(y-R_{n} y\right)^{(i)}(x)=\int_{0}^{x} E_{i}(x, t) w(t) v_{n}(t) d t, \quad 0 \leqslant x \leqslant b, 0 \leqslant i \leqslant s-1$.

Let $x$ be arbitrary in $[0, b]$ and suppose $x \in\left[x_{v-1}, x_{v}\right)$ for some $v$, and define

$$
F_{l}=\int_{x_{l-1}}^{x_{l}} E_{i}(x, t) w(t) v_{n}(t) d t, \quad 1 \leqslant l \leqslant v .
$$

For each $l=1,2, \ldots, v$, we can assume that $v_{n} \in C^{\tilde{p}+r+1}\left[x_{l-1}, x_{l}\right]$ using the limiting values of $v_{n}$ in (8.12). Hence, from (8.12) and the definition of $P_{n}$ we see that

$$
v_{n}^{(u)}\left(\gamma_{k l}\right)=0, \quad 1 \leqslant k \leqslant p, 0 \leqslant u \leqslant r_{k}, 1 \leqslant l \leqslant v
$$

Therefore, we can write

$$
F_{l}=\int_{x_{l-1}}^{x_{l}} h_{x}(t) \prod_{k=1}^{p}\left(t-\gamma_{k l}\right)^{1+r_{k}} d t, \quad 1 \leqslant l \leqslant v
$$


where

$$
h_{x}(t)=E_{i}(x, t) w(t) v_{n}\left[\frac{\gamma_{1 l}, \ldots, \gamma_{1 l}}{1+r_{1}}, \ldots, \frac{\gamma_{p l}, \ldots, \gamma_{p l}}{1+r_{p}}, t\right] .
$$

The last expression involving $v_{n}$ is the $\tilde{p}$ th divided difference of $v_{n}$ on $t$ and on the points $\gamma_{k l}$ with multiplicities $1+r_{k}$. If we write for $t \in\left[x_{l-1}, x_{l}\right]$,

$$
\begin{aligned}
h_{x}(t)= & \sum_{u=0}^{r} h_{x}^{(u)}\left(x_{l-1}\right) \frac{\left(t-x_{l-1}\right)^{u}}{u !} \\
& +h_{x}^{(r+1)}\left(\xi_{t}\right) \frac{\left(t-x_{l-1}\right)^{r+1}}{(r+1) !}, \quad x_{l-1}<\xi_{t}<x_{l},
\end{aligned}
$$

then

(8.15) $F_{l}=\int_{x_{l-1}}^{x_{l}} \prod_{k=1}^{p}\left(t-\gamma_{k l}\right)^{1+r_{k}} \frac{\left(t-x_{l-1}\right)^{r+1}}{(r+1) !} h_{x}^{(r+1)}\left(\xi_{t}\right) d t, \quad 1 \leqslant l \leqslant v$.

But

$$
\begin{array}{r}
h_{x}^{(r+1)}(t)=\sum_{u=0}^{r+1}\left(\begin{array}{c}
r+1 \\
u
\end{array}\right)\left(E_{i}(x, \cdot) w\right)^{(r+1-u)}(t) v_{n}^{(\tilde{p}+u)}\left(z_{u, t}\right) \frac{u !}{(\tilde{p}+u) !} \\
x_{l-1}<t, z_{u, t}<x_{l} .
\end{array}
$$

Hence, for some $C$ independent of $x$ and $n$,

$$
\sup _{x_{l-1}<t<x_{l}}\left|h_{x}^{(r+1)}(t)\right| \leqslant C \sup _{x_{l-1}<t<x_{l}} \sum_{u=0}^{r+1}\left|v_{n}^{(\tilde{p}+u)}(t)\right| .
$$

Now from (8.12) it follows by (8.2), (8.7) and Lemma 5.4 that for some $C$ independent of $l$,

$$
\sup _{x_{l-1}<t<x_{l}}\left|v_{n}^{(i)}(t)\right| \leqslant C\left(\frac{\left|\Delta_{n}\right|}{\Delta x_{l}}\right)^{\tilde{p}}, \quad 0 \leqslant i \leqslant \tilde{p}+r+1,0 \leqslant l \leqslant v
$$

This bound and (8.15) imply that for some $C$ independent of $n$ and $x$,

$$
\left|F_{l}\right| \leqslant C\left|\Delta_{n}\right|^{\tilde{p}}\left(\Delta x_{l}\right)^{r+2}, \quad 0 \leqslant l \leqslant v .
$$

Write (8.13) as

$$
\left(y-R_{n} y\right)^{(i)}(x)=\sum_{l=1}^{v-1} F_{l}+\int_{x}^{x} h_{x-1}(t) \prod_{k=1}^{p}\left(t-\gamma_{k v}\right)^{1+r_{k}} d t
$$

If $x=x_{v-1}$, then (8.17) and (8.18) imply 


$$
\left(y-R_{n} y\right)^{(i)}\left(x_{v-1}\right)=O\left(\left|\Delta_{n}\right|^{\tilde{p}+r+1}\right), \quad 0 \leqslant i \leqslant s-1 .
$$

If $x_{v-1}<x<x_{v}$, then since $E_{i}(x, t)$ has an $(s-1-i)$ th-fold zero at $x=t$, (8.16), (8.18) and the definition of $h_{x}(t)$ imply

$$
\begin{aligned}
\left(y-R_{n} y\right)^{(i)}(x) & =O\left(\left.\Delta_{n}\right|^{\tilde{p}+r+1}\right)+O\left(\left|\Delta_{n}\right|^{\tilde{p}+s-i}\right) \\
& =O\left(\mid \Delta_{n} \tilde{p}^{\tilde{p}+\min (r+1, s-i)}\right), \quad 0 \leqslant i \leqslant s-1 .
\end{aligned}
$$

The conclusions of the theorem are now obtained from (8.3), (8.19) and (8.20), since $r+1 \leqslant \tilde{p}$.

Suppose $r_{k}=0,1 \leqslant k \leqslant p$. Then (8.1) is satisfied by the Gaussian points if $r=p-1$, by the Radau points if $r=p-2$, and by the Lobatto points if $r=p-3$. Turán [25] showed that if all $r_{k}$ are equal to some fixed even positive integer, then (8.1) is satisfied for $r=p-1$ by the zeros of a certain polynomial; Stroud and Stancu [24] tabulated some of these "multiple Gaussian points." Stancu and Stroud [23] showed that one can fix $n_{1}$ of the $p$ points $\gamma_{k}$, assign them arbitrary multiplicities, and find the remaining $p-n_{1}$ points of multiplicity one to satisfy (8.1) for $r=p-n_{1}-1$; they also tabulated some such points for several choices of the fixed points. Wittenbrink [28] has investigated methods which use these points, with the restriction that only the endpoints can have multiplicities larger than one. Our theory allows a more general choice of points and multiplicities, but the highest chosen multiplicity must not exceed $s-1-m$.

9. Extensions of Methods Considered in the Literature. The idea of constructing global approximations more general than piecewise polynomials can be useful if the differential equation exhibits characteristics which can be exploited. For example, we could let

$$
a_{k}=\frac{\partial f}{\partial y^{(k)}}\left(0, y(0), \ldots, y^{(m)}(0)\right),
$$

for those $k$ for which $\partial f / \partial y^{(k)}$ does not vary much in $[0, b]$. Our theory assumes that $w$ and the $a_{k}$ are constant throughout the numerical process. However, we can change them after $y_{n}$ has been found in an interval $[0, a]$ and consider a new problem in $[a, b]$ with the initial values given by $y_{n}$. The asymptotic error rate is the same throughout $[0, b]$, as can be seen in Corollary 4.1 .

Ixaru [13] approximates the coefficients and inhomogeneous term of second order linear differential equations by step functions, then solves the resulting equation exactly; the method is of low order but it is explicit. Pruess [18] obtained methods of arbitrarily high order by replacing the coefficients and inhomogeneous term of an $n$th order equation by piecewise polynomials, then using Taylor series techniques to solve the resulting problem. Cooper [6] used a weight function but did not consider the global approximations.

For the remainder of this section we will assume that $a_{k}=0,0 \leqslant k \leqslant m, w \equiv$ 1 and $g_{i}^{n}=y^{(i)}(0), 0 \leqslant i \leqslant s-1$, and discuss how particular choices of $P_{n}$ reduce 
our methods to methods already considered by several authors to deal with (2.1), (2.2).

If $P_{n}$ is the operator of Example 5.1 and $r_{k}=0,1 \leqslant k \leqslant p$, then we obtain the method of collocation with piecewise polynomials [7], [10], [11], [19], [28] . If in addition $\gamma_{k}=(k-1) /(p-1), 1 \leqslant k \leqslant p$, then we obtain for $s=1$ the NewtonCotes block implicit discrete methods of Watts and Shampine [26], some of whose global aspects were investigated by Callender [5] and Williams and de Hoog [27]; for $s=2$, we have the spline methods of Micula [17]. All of these methods are equivalent to collocation with piecewise polynomials; notice that if $p$ is odd, Eq. (8.1) holds for $r=0$ so that higher rates are obtained.

Wittenbrink [28] has considered $\gamma_{1}=0, \gamma_{p}=1, r_{1}=r_{p}, r_{p}$ arbitrary, but $r_{i}$ $=0$ for $2 \leqslant i \leqslant p-1$. We have considered arbitrary choices of $\gamma_{k}$ and $r_{k}$ as long as $\max r_{k} \leqslant s-m-1$, and have obtained higher rates along the lines of [7], even if some $a_{k} \neq 0$ or $w \neq \equiv 1$. Moreover, the theory presented here can also be applied if $\gamma_{1}=0, \gamma_{p}=1,0 \leqslant r_{p}-r_{1} \leqslant 1, r_{p}$ arbitrary, and $0 \leqslant r_{i} \leqslant r_{p}$ for $2 \leqslant i \leqslant p-1$; these conditions give rise to some $\boldsymbol{A}$-stable methods for the numerical solution of first order problems. Details appear in [15] and will be presented elsewhere.

If $P_{n}$ is derived from the operators $Q_{2}$ of Example 5.2 with $l=p$, one has the natural spline block implicit methods of Andria, Byrne and Hill [2] for first order problems; global approximations were not considered in [2] .

Several of the projections of [28] satisfy Lemma 5.1, but we emphasize the fact that the operators $Q$ of Section 5 are not necessarily projections.

Finally, we point out that some of the techniques presented here can be used to obtain error estimates for derivatives higher than $s-1$. Some such results are presented in Theorem 7.1.

10. Sample Calculations. In the following tables we present results which illustrate the rates of convergence and the improvement caused by choosing some $a_{k}$ $\neq 0$ or $w \neq \equiv 1$. All calculations were carried out on a CDC 6400 in single precision (which is approximately fourteen decimal digits). The column next to each column of errors represents the computed orders of convergence

$$
\frac{\log \left(E_{n}\left(h_{1}\right) / E_{n}\left(h_{2}\right)\right)}{\log \left(h_{1} / h_{2}\right)}
$$

based on successive mesh sizes $h_{1}$ and $h_{2} . E_{n}$ is a discrete or continuous error norm, as defined in each example. All the methods are based on the operators of Example 5.1, and we used the alternate approach described in Section 6. The functions $F_{n, j}^{r}$ and $d_{i j}$ in (6.7) and their derivatives were found explicitly in terms of the $a_{k}$ and evaluated at the $\gamma_{k j}$ in advance (as required) to produce the linear system for the unknowns $y_{n, r+1}^{(v)}\left(\gamma_{k j}\right)$. If $\Delta x_{j}$ is constant, the matrix of the system is the same from step to step.

Example 10.1. To illustrate the use of a weight function consider

$$
y^{\prime}(x)=\sqrt{x} y(x), \quad 0 \leqslant x \leqslant 1, y(0)=1 .
$$


The approximations $y_{n}$ are obtained using $P_{n}$ with $p=2, \gamma_{1}=0, \gamma_{2}=1$, $r_{1}=0, r_{2}=0$. If $w \equiv 1$, this amounts to collocation with piecewise quadratics. If $w(x)=\sqrt{x}$, we obtain a new method. The results are shown in Table 1 , where

$$
E_{n}^{(i)}=\sup _{0<x<1}\left|\left(y-y_{n}\right)^{(i)}(x)\right|, \quad i=0,1 \text {, and } 5.22(-2) \text { stands for } 5.22 \times 10^{-2} .
$$

Not only is $E_{n}^{(i)}$ smaller for the new method, but also the new method converges faster.

TABLE 1

Use of a weight function

\begin{tabular}{|c|c|c|c|c|c|c|c|c|}
\hline \multirow[b]{2}{*}{$\Delta \mathbf{x}_{j}$} & \multicolumn{4}{|c|}{ Collocation } & \multicolumn{4}{|c|}{ New Method } \\
\hline & $E_{n}$ & & $\mathrm{E}_{\mathrm{n}}^{(1)}$ & & $E_{n}$ & & $E_{n}^{(I)}$ & \\
\hline $1 / 2$ & $5.22(-2)$ & & $1.51(-1)$ & & $3.79(-2)$ & & $8.04(-2)$ & \\
\hline $1 / 4$ & $2.59(-2)$ & 1.01 & $1.19(-1)$ & 0.34 & $9.26(-3)$ & 2.03 & $2.46(-2)$ & 1.71 \\
\hline $1 / 8$ & $1.18(-2)$ & 1.13 & $8.62(-2)$ & 0.47 & $2.30(-3)$ & 2.01 & $6.95(-3)$ & 1.82 \\
\hline $1 / 16$ & $4.80(-3)$ & 1.30 & $6.16(-2)$ & 0.48 & $5.72(-4)$ & 2.01 & $1.86(-3)$ & 1.90 \\
\hline $1 / 32$ & $1.86(-3)$ & 1.37 & $4.37(-2)$ & 0.50 & $1.43(-4)$ & 2.00 & $4.82(-4)$ & 1.95 \\
\hline
\end{tabular}

Example 10.2. Consider

$$
y^{\prime}(x)=(-5+x) y(x), \quad 0 \leqslant x \leqslant 4, y(0)=1
$$

We have used $P_{n}$ with $p=3, \gamma_{1}=0, \gamma_{2}=0.5, \gamma_{3}=1, r_{1}=r_{2}=r_{3}=0$. If $a_{0}=0$ and $w \equiv 1$, the method is collocation with piecewise cubics. We have also taken $w \equiv$ 1 and $a_{0}=-4$ for the interval $[0,2]$, and $w \equiv 1$ and $a_{0}=-2$ for the interval $[2,4]$. Tables 2 and 3 show the results obtained. Notice that the rates of convergence are about the same. The errors here are defined by

$$
E_{n}^{(i)}=\max _{1<j<n}\left|\left(y-y_{n}\right)^{(i)}\left(x_{j}^{-}\right)\right|, \quad i=0,1,2,3
$$

TABLE 2

Collocation with piecewise cubics

\begin{tabular}{|l|cl|cc|cc|cc|}
\hline$\Delta x_{j}$ & $E_{n}$ & & $E_{n}^{(1)}$ & $E_{n}^{(2)}$ & $E_{n}^{(3)}$ \\
\hline $1 / 4$ & $1.46(-3)$ & & $6.96(-3)$ & & $2.06(0)$ & & $3.97(1)$ & \\
$1 / 8$ & $8.56(-5)$ & 4.09 & $4.07(-4)$ & 4.10 & $7.13(-1)$ & 1.53 & $3.06(1)$ & 0.38 \\
$1 / 16$ & $5.47(-6)$ & 3.97 & $2.63(-5)$ & 3.95 & $2.12(-1)$ & 1.75 & $1.92(1)$ & 0.67 \\
$1 / 32$ & $3.41(-7)$ & 4.00 & $1.64(-6)$ & 4.00 & $5.78(-2)$ & 1.87 & $1.08(1)$ & 0.83 \\
\hline
\end{tabular}


TABLE 3

Collocation with modified functions

\begin{tabular}{|l|cl|cl|ll|ll|}
\hline$\Delta x_{j}$ & $E_{n}$ & & $E_{n}^{(1)}$ & $E_{n}^{(2)}$ & $E_{n}^{(3)}$ & \\
\hline $1 / 4$ & $2.86(-4)$ & & $1.36(-3)$ & & $4.66(-1)$ & & $7.98(0)$ & \\
$1 / 8$ & $1.72(-5)$ & 4.06 & $8.37(-5)$ & 4.02 & $1.80(-1)$ & 1.37 & $7.35(0)$ & 0.12 \\
$1 / 16$ & $1.12(-6)$ & 3.94 & $5.41(-6)$ & 3.95 & $5.64(-2)$ & 1.67 & $5.00(0)$ & 0.56 \\
$1 / 32$ & $7.01(-8)$ & 4.00 & $3.38(-7)$ & 4.00 & $1.58(-2)$ & 1.84 & $2.92(0)$ & 0.78 \\
\hline
\end{tabular}

For this choice of $\gamma_{k}$ and $r_{k}$, Eq. (8.1) is satisfied for $r=0$, hence the higher rates for $E_{n}$. The higher rates for $E_{n}^{(1)}$ are, for example, because of

$$
\left(y_{n}-y\right)^{\prime}\left(x_{j}\right)=P_{n}\left((-5+(\cdot)) y_{n}\right)\left(x_{j}\right)-\left(-5+x_{j}\right) y\left(x_{j}\right)=\left(-5+x_{j}\right)\left(y_{n}-y\right)\left(x_{j}\right) .
$$

Example 10.3. It is known [9] that a differential equation of the form

$$
y^{\prime \prime}(x)=-k^{2}(1+p(x)) y(x), \quad a \leqslant x,
$$

where $k$ is a positive constant and $\int_{a}^{\infty} p(x) d x<\infty$ has solutions of the form

$$
y(x)=c_{1} \cos (k x)+c_{2} \sin (k x)+o(1)
$$

for large $x$. Hence we expect particularly favorable results if we choose $w \equiv 1, a_{0}=$ $-k^{2}, a_{1}=0$. As an illustration, we approximated the solution of Bessel's equation

$$
y^{\prime \prime}(x)=\left(-100-1 / 4 x^{2}\right) y(x), \quad 1 \leqslant x \leqslant 6,
$$

with initial values chosen so that the solution is $y(x)=\sqrt{x} J_{0}(10 x)$. Table 4 shows errors at several values of $x$ for $\Delta x_{j}=0.02$. Here $E_{n}^{(i)}=\left|\left(y-y_{n}\right)^{(i)}\left(x^{-}\right)\right|, i=0,1,2$.

\section{TABLE 4}

\section{Comparison of collocation methods}

\begin{tabular}{|c|l|l|l|l|l|l|}
\hline \multirow{2}{*}{$x$} & \multicolumn{3}{|c|}{ Piecewise Cubics } & \multicolumn{3}{c|}{ Modified Functions } \\
\cline { 2 - 7 } & $E_{\mathrm{n}}$ & $\mathrm{E}_{\mathrm{n}}^{(1)}$ & $\mathrm{E}_{\mathrm{n}}^{(2)}$ & $\mathrm{E}_{\mathrm{n}}$ & $\mathrm{E}_{\mathrm{n}}^{(1)}$ & $\mathrm{E}_{\mathrm{n}}^{(2)}$ \\
\hline 2 & $3.8(-7)$ & $1.1(-5)$ & $8.1(-2)$ & $4.7(-10)$ & $1.9(-8)$ & $4.7(-5)$ \\
4 & $2.9(-6)$ & $6.2(-6)$ & $1.9(-2)$ & $2.6(-9)$ & $4.1(-9)$ & $6.1(-7)$ \\
6 & $2.2(-6)$ & $4.0(-5)$ & $7.2(-2)$ & $1.5(-9)$ & $2.5(-8)$ & $5.1(-6)$ \\
\hline
\end{tabular}

We have taken $p=2, \gamma_{1}=\left(1-(1 / 3)^{1 / 2}\right) / 2, \gamma_{2}=1-\gamma_{1}$ (Gaussian points), $r_{1}=r_{2}$ $=0$, and $k=10$. Also shown are the results for $k=0$ (collocation with piecewise cubics).

Example 10.4. Our last example is the nonlinear problem

$$
y^{\prime \prime}(x)=2(y(x))^{2}\left(4 x^{2} y(x)-1\right), \quad 0 \leqslant x \leqslant 1, y(0)=1, y^{\prime}(0)=0 .
$$


We have taken $a_{0}=a_{1}=0, w \equiv 1$, and the operators $P_{n}$ of Example 5.1 with $p=2$, $\gamma_{1}=0, \gamma_{2}=1, r_{1}=0, r_{1}=1$. The results are shown in Table 5, where

$$
\begin{aligned}
& E_{n}^{(i)}=\max _{0<x<1}\left|\left(y_{n}-y\right)^{(i)}(x)\right|, \quad i=0,1,2, \\
& E_{n}^{(i)}=\max _{1<j<n x_{j-1}<x<x_{j}} \sup _{x_{j}}\left|\left(y_{n}-y\right)^{(i)}(x)\right|, \quad i=3,4 .
\end{aligned}
$$

TABLE 5

\begin{tabular}{|c|c|c|c|c|c|c|c|c|c|c|}
\hline$\Delta x_{j}$ & $E_{n}$ & & $E_{n}^{(1)}$ & & $E_{n}^{(2)}$ & & $E_{n}^{(3)}$ & & $E_{n}^{(4)}$ & \\
\hline $1 / 4$ & $7.08(-3)$ & & $1.17(-2)$ & & $3.03(-2)$ & & $7 \cdot 39(-1)$ & & $1.25(1)$ & \\
\hline $1 / 8$ & $8.08(-4)$ & 3.13 & $1.44(-3)$ & 3.02 & $4.57(-3)$ & 2.73 & $2.47(-1)$ & 1.58 & $7.64(0)$ & 0.71 \\
\hline $1 / 16$ & $9.71(-5)$ & 3.06 & $1.78(-4)$ & 3.02 & $5.88(-4)$ & 2.96 & $6.49(-2)$ & 1.93 & $4.14(0)$ & 0.88 \\
\hline $1 / 32$ & $1.19(-5)$ & 3.01 & $2.22(-5)$ & 3.00 & $7 \cdot 34(-5)$ & 3.00 & $1.63(-2)$ & 1.99 & $2.09(0)$ & 0.99 \\
\hline $1 / 64$ & $1.48(-6)$ & 3.01 & $2.77(-6)$ & 3.00 & $9.21(-6)$ & 2.99 & $4.08(-3)$ & 2.00 & $1.04(0)$ & 1.01 \\
\hline $1 / 128$ & $1.84(-7)$ & 3.01 & $3.46(-7)$ & 3.00 & $1.15(-6)$ & 3.00 & $1.02(-3)$ & 2.00 & $5.23(-1)$ & 0.99 \\
\hline
\end{tabular}

A piecewise polynomial approximation

Department of Mathematics

Emory University

Atlanta, Georgia 30322

1. J. H. AHLBERG, E. N. NILSON \& J. L. WALSH, "Convergence properties of generalized splines," Proc. Nat. Acad. Sci. U.S.A., v. 54, 1965, pp. 344-350.

2. G. D. ANDRIA, G. D. BYRNE \& D. R. HILL, "Natural block implicit methods," BIT, v. 13, 1973, pp. $131-144$.

3. P. M. ANSELONE, Collectively Compact Operator Approximation Theory and Applications to Integral Equations, Prentice-Hall, Englewood Cliffs, N. J., 1971.

4. G. BIRKHOFF, M. H. SCHULTZ \& R. S. VARGA, "Piecewise Hermite interpolation in one and two variables with applications to partial differential equations," Numer. Math., v. 11, 1968, pp. $232-256$.

5. E. D. CALLENDER, "Single step methods and low order splines for solutions of ordinary differential equations," SIAM J. Numer. Anal., v. 8, 1971, pp. 61-66.

6. G. J. COOPER, "Interpolation and quadrature methods for ordinary differential equations," Math. Comp., v. 22, 1968, pp. 69-76.

7. C. R. DE BOOR \& B. SWARTZ, "Collocation at Gaussian points," SIAM J. Numer. Anal., v. 10,1973 , pp. $582-606$.

8. N. DUNFORD \& J. T. SCHWARTZ, Linear Operators, Interscience, New York, 1957.

9. W. GAUTSCHI, "Numerical integration of ordinary differential equations based on trigonometric polynomials," Numer. Math., v. 3, 1961, pp. 381-397.

10. H. L. HULME, "Discrete Galerkin and related one-step methods for ordinary differential equations," Math. Comp., v. 26, 1972, pp. 881-891.

11. H. L. HULME, "One-step piecewise polynomial Galerkin methods for initial value problems," Math. Comp., v. 26, 1972, pp. 415-426.

12. E. ISAACSON \& H. B. KELLER, Analysis of Numerical Methods, Wiley, New York, 1966.

13. L. GR. IXARU, "A new method for solving inhomogeneous second order differential equations," Rev. Roumaine Math. Pures Appl., v. 19, 1974, pp. 199-203. 
14. D. JACKSON, The Theory of Approximation, Amer. Math. Soc. Colloq. Publ., vol. 12, Amer. Math. Soc., Providence, R. I., 1930.

15. L. KRAMARZ, Global Approximations to Solutions of Initial Value Problems, Ph.D. Thesis, Georgia Inst. of Tech., Atlanta, 1977.

16. T. LYCHE \& L. L. SCHUMAKER, Local Spline Approximation Methods, Tech. Summary Report 1417, Math. Res. Center, U. S. Army, Univ. of Wisconsin, Madison, 1974.

17. GH. MICULA, "The numerical solution of nonlinear differential equations by deficient spline functions," $Z$. Angew. Math. Mech., v. 55, 1975 , pp. T.254-T.256.

18. S. A. PRUESS, "Solving linear boundary value problems by approximating the coefficients," Math. Comp., v. 27, 1973, pp. 551-561.

19. R. D. RUSSELL \& L. F. SHAMPINE, "A collocation method for boundary value problems," Numer. Math., v. 19, 1972, pp. 1-28.

20. I. J. SCHOENBERG, "On monosplines of least deviation and best quadrature formulae," SIAM J. Numer. Anal., v. 2, 1965, pp. 144-170.

21. M. H. SCHULTZ \& R. S. VARGA, "L-splines," Numer. Math., v. 10, 1967, pp. 345369.

22. A. SPITZBART, “A generalization of Hermite's interpolation formula," Amer. Math. Monthly, v. 67, 1960 , pp. 42-46.

23. D. D. STANCU \& A. H. STROUD, "Quadrature formulas with simple Gaussian nodes and multiple fixed nodes," Math. Comp., v. 17, 1963, pp. 384-394.

24. A. H. STROUD \& D. D. STANCU, "Quadrature formulas with multiple Gaussian nodes," SIAM J. Numer. Anal., v. 2, 1965, pp. 129-143.

25. P. TURÁN, "On the theory of the mechanical quadrature," Acta Sci. Math. (Szeged), v. 12, 1950, pp. 30-37.

26. H. A. WATTS \& L. F. SHAMPINE, " $A$-stable block implicit one-step methods," $B I T$, v. 12, 1972 , pp. $252-266$.

27. J. WILLIAMS \& F. DE HOOG, "A class of $A$-stable advanced multistep methods," Math. Comp., v. 28, 1974, pp. 163-177.

28. K. A. WITTENBRINK, "High order projection methods of moment and collocation type for nonlinear boundary value problems," Computing, v. 11, 1973, pp. 255-274. 